\title{
PRODUCCIÓN DE ARRABIO CON MINERAL DE HIERRO DE TACNA, ESTUDIO PRELIMINAR
}

Carlos Vivar Colquicocha'; Zoilo Valdivia Infantas ${ }^{2}$

\begin{abstract}
$\boldsymbol{R} \boldsymbol{E} \boldsymbol{S} \boldsymbol{U} \boldsymbol{M} \boldsymbol{E}$
El presente trabajo permite determinar la tecnología más adecuada para producir arrabio en condiciones específicas como: planta de pequeña capacidad, baja inversión y costos razonables, aprovechando ventajas comparativas como contar con un mineral de hierro de buena calidad, disponibilidad de un carbón de caracteristicas aceptables, para la producción de arrabio utilizando directamente el mineral de hierro y el carbón, es decir, sin requerir que el mineral sea procesado previamente mediante la concentración y producción de pellets, que es la carga característica de los altos hornos, e igualmente sin requerir que el carbón sea acondicionado como coque, condiciones que hacen un proyecto rentable a pesar de su pequeña capacidad de producción.

El presente estudio también tiene por objetivo determinar si la rentabilidad del proyecto es atractiva y justifica la elaboración de un estudio de factibilidad.

\section{ABSTRACT}

The present work allows to determine the technology to produce pig-iron under specific conditions as: Plant of small capacity, low investment and reasonable costs, taking advantage as: good quality mineral iron and acceptable coal, for the pig-iron production using the iron mineral and the coal directly, that is to say without requiring that the mineral is processed previously by means of the concentration and pellets production that it is the characteristic load of the high ovens and equally without requiring that the coal is conditioned as coke, all of that make a profitable project in spite of its small production capacity.
\end{abstract}

The present work also has for objective to determine if the profitability of the project is attractive and it justifies the elaboration of a study of feasibility.

\section{INTRODUCCIÖN}

En la zona de Sama - Tacna, se cuenta con mineral de hierro con un contenido de $58 \%$, de hierro, calificado como de alta ley y bajo azufre que hace posible cargarlo al horrio sin ningún tratamiento previo y también se cuenta con un carbón bituminoso en actual uso en la Central Térmica de llo y que puede también ser usado sin tratamiento previo, condiciones que permitiría explotar el yacimiento de mineral de hierro de la zona de Sama y de otras que tengan caracteristicas similares en el país.

\section{OBJETIVO DE LA INVESTIGACIÓN}

Realizar pruebas con mineral de la zona de Tacna y carbón colombiano que se encuentra disponible en la Central Térmica de llo. El carbón es bituminoso con $35 \%$ de contenido de materias volátiles y será usado sin ninguna preparación previa a no ser el ajuste a una granulometría de $11 / 2^{\prime \prime}$. Estas pruebas servirán para demostrar que se puede producir arrabio con mineral directo de mina ajustado a una granulometria de $1^{n}$, de modo de obtener algunos parámetros de operación que puedan servir para escalar a un proceso industrial, permitiendo la elaboración de un estudio preliminar de factibilidad.

\section{ESTUDIO DE MERCADO}

El estudio de mercado indica que es factible colocar en el mercado nacional $5000 \mathrm{t}$ de arrabio en las aproximadamente 50 principales fundiciones del país a un promedio de 5 a 30 t mensuales y a un precio de $230 \$$ por tonelada de arrabio.

También indica que es factible exportar la diferencia de la producción, es decir 4620 t de arrabio a países de Latinoamérica, Europa o Asia, debido a que hay un déficit de oferta a nivel internacional como 
consecuencia de la insuficiente producción de Coque y que ha motivado que China inicie un gran proyecto de producción de Coque que estará operativo dentro de 3 años y que será insuficiente para revertir la situación, por lo que es probable que el precio del arrabio se mantenga por encima de los 230 \$ por tonelada dentro de los próximos 5 años. Esto es previsible porque en los próximos años se va a producir una aceleración en el consumo de chatarra que ocasionará que esta ya no sea un gran competidor del arrabio, incluso en las economlas menos desarrolladas por lo menos durante los próximos 5 años.

\section{ESTUDIO TÉCNICO}

El estudio técnico indica que el mineral es fácilmente reducible y de bajo contenido de azufre, por lo que el arrabio producido será de buena calidad.

El mineral a emplearse tiene una ley de $58 \%$ de hierro, $4 \%$ de silice, $0,017 \%$ de azufre y $0,085 \%$ de fósforo.

El carbón que se empleará es de bajo contenido de azufre, solo que presenta un alto contenido de materias volátiles $38,35 \%$ que ocasiona un mayor consumo unitario con respecto al coque y una menor eficiencia térmica del orden del $40 \%$, pero que está compensado por su menor precio, además es posible mejorar la eficiencia térmica mediante proyectos adicionales a ejecutarse en los años siguientes.

\section{CONCLUSIONES DE LAS PRUEBAS REALIZADAS}

1. El carbón a emplearse sería el colombiano $C M, O$ cualquier otro carbón de caracteristicas similares, las cuales son: M.V.: $36,52 \%$, Cenizas $5 \%$, azufre: 0,65 $\%$.

2. Este carbón se coquiza dentro del horno y se quema en forma similar al coque, produciendo una adecuada combustión y elevación de la temperatura a la altura de las toberas, llegándose a la temperatura crítica necesaria para producir arrabio del orden de $1600^{\circ} \mathrm{C}$.

3. El inconveniente de este carbón el de tener un alto contenido de materias volátiles, por lo que el rendimiento de este carbón con respecto al coque es del orden del $51 \%$, considerando el contenido de carbono fijo, lo que origina que se tenga que considerar un parámetro de consumo mayor, es decir: $1.10 \mathrm{Kg}$. Carbón / Kg. arrabio.

\section{INGENIERIA DEL PROYECTO}

\section{UBICACIÓN DEL PROYECTO}

MINA: La mina se encuentra ubicada en el distrito de Sama, provincia de Tacna, departamento de Tacna, a $25 \mathrm{Km}$. al NE de la ciudad de Tacna.

PLANTA: La Planta se encuentra ubicada en el distrito de Pachia, provincia de Tacna, departamento de Tacna, a $48 \mathrm{Km}$. al NO de la ciudad de Tacna.

\section{EQUIPOS PRINCIPALES}

El proceso de producción de arrabio a partir de mineral de hierro, empleando carbón bituminoso de alto contenido de materias volátiles, se realizará con los siguientes equipos principales:

\section{ALTO HORNO}

Este equipo permitirá la reducción del mineral de hierro, que consiste en la eliminación del oxígeno mediante el monóxido de carbono que se produce por la combustión del carbón a la altura de las toberas por donde ingresa el aire precalentado a $700^{\circ} \mathrm{C}$. Además se realiza la fusión del mineral y la incorporación del carbón dentro del metal hierro produciéndose asi el arrabio que se cuela a $1400^{\circ} \mathrm{C}$.

\section{INTERCAMBIADOR DE CALOR}

Sirve para transferir el calor de los gases calientes que salen del horno al aire frío que se produce con el soplante, elevándose la temperatura del aire a $700^{\circ} \mathrm{C}$.

\section{SEPARADOR DE SÓLIDOS}

Permite eliminar las partículas sólidas de los gases del horno para evitar la expulsión de estas partículas a los alrededores de la Planta, controlándose asi la contaminación del medio ambiente.

\section{SOPLANTE}

Este equipo es el que produce el aire frío que es precalentado en el intercambiador y que suministra oxígeno necesario para la combustión del carbón a la altura de las toberas del horno.

\section{CHANCADORA DE QUIJADAS}

Este equipo permitirá triturar tanto el mineral como el fundente para ajustarlos a la granulometria de $11 / 2$ que requiere el proceso. 


\section{ESTUDIO ECONÓMICO FINANCIERO}

La inversión es de $500000 \$$, desagregada en la iguiente forma:

$\begin{array}{ll}\text { Préstamo Bancario } & : 360000 \$ \\ \text { Aporte propio } & : 140000 \$\end{array}$

Los ingresos son del orden de $2484000 \$$ / año, el costo de producción del orden de $1853000 \$$ / año, or lo que la utilidad bruta es de $631000 \$$ / año, jenerándose una utilidad antes de impuestos de 545 $00 \$$ / año y una utilidad neta de $381600 \$$ / año y de $\$ 20700$ a partir del sexto año.

En el flujo de Caja se tiene un flujo económico de $\$ 24000 \$$ / año y un flujo financiero de 509700 \$ / aก̃o.

\section{EVALUACIÓN DEL PROYECTO}

La rentabilidad del proyecto medida por la Tasa Interna de Retorno Económica es $64 \%$ que permite una recuperación de la inversión en dos años, por lo que el proyecto es muy bueno. Las condiciones financieras del proyecto medidas por la Tasa Interna de Retorno Financiera son de $70 \%$, lo cual indica que las condiciones del préstamo son recomendables.

\section{CONCLUSIONES}

1. Como se ha indicado en la introducción, es posible producir arrabio utilizando mineral y carbón directos de mina, solo con el ajuste de la granulometría.

2. El arrabio producido hasta la fecha tiene la composición siguiente:

Fe: $93.30 \%$, C: $3.30 \%$, Si: $0.6 \%$, S: $0.37 \%$, P: $0.28 \%$.

3. El contenido de azufre es alto, por lo que por ahora constituye un producto para usos no muy exigentes; sin embargo, es posible obtener un contenido más bajo de azufre del orden de $0.20 \%$ para poder emplearlo en producción de fundiciones. La razón principal de no haber alcanzado bajos porcentajes de azufre radica en no haber alcanzado una mayor temperatura a la altura de las toberas por no haber logrado calentar el aire a más de $300^{\circ} \mathrm{C}$.

4. Las pruebas no dan directamente el consumo unitario de carbón, el cual tiene que ser escalado, esto como consecuencia del tamaño pequeño del horno y a que aún no está acondicionado para pruebas continuas de más de una semana, en cuyo caso se podría tener un parámetro aproximado a la realidad; sin embargo, se ha escalado un consumo de 1,17 toneladas de carbón por tonelada de arrabio, parámetro que se ha considerado para el diseño de la planta industrial.

5. El factor crítico para lograr una aceptable rentabilidad lo constituye el precio del carbón y su adaptabilidad al proceso.

6. El carbón antracita no es aceptable por su alto precio y por requerir inyección de finos

7. El presente proyecto, al tener un periodo de recuperación de la inversión de dos años, se le califica de muy bueno. Esto se ha hecho posible por el incremento del precio del acero, arrabio y de la chatarra ocurrido desde el segundo semestre del año 2003 y que se espera se mantenga en estos niveles durante los próximos 3 a 5 años.

8. También es consecuencia de contar con un mineral de alta ley y de bajo contenido de azufre y fósforo y de contar con un carbón de bajo contenido de azufre procedente de Colombia y que está disponibie en la zona de Tacna por utilizarse para la generación de energía en la Planta Térmica de lio.

9. La venta del producto no debe ofrecer mayores problemas porque se trata de un mercado nacional que se encuentra desabastecido de arrabio y de chatarra y de un mercado internacional con déficit de oferta, por lo que la exportación tampoco debe afrontar dificultades, sobre todo tratándose de un arrabio de bajo contenido de azufre y fósforo.

\section{REFERENCIA BIBLIOGRÁFICA}

1. H.M. Boyiston: Introducción a la Metalurgia del Hierro y del Acero Ed. Wiley USA 1990.

2. Paráis Barreiro, Josó; Fabricación del Hierro Acero y Fundiciones, Edit. Ourmo S.A. España, 1990.

3. J.S. Hirkaldy and R.G. Ward, Aspects of Modern Ferrous Metallurgy, Edición, 1984.

4. H. Appold K Feller, Tecnología de los metales, Edit Reverté, España 1994.

5. Capelani R. Diversificación de combustibles utilisables au furneau, Paris, 1975.

6. Paoletti , R. Degradación mecánica del coque, revista tecnica Paris, 1975.

7. Coche, L. Enriquecimiento de los minerales de Hierro IRSID Paris 1974. 